\title{
Interplay between temperature and bandgap energies on the outdoor performance of perovskite/silicon tandem solar cells
}

Erkan Aydin ${ }^{1 * \dagger}$, Thomas G. Allen ${ }^{1 \dagger}$, Michele De Bastiani ${ }^{1}$, Lujia Xu ${ }^{1}$, Jorge Ávila ${ }^{1,2}$, Michael Salvador ${ }^{1}$, Emmanuel Van Kerschaver ${ }^{1}$, Stefaan De Wolf ${ }^{1 *}$

${ }^{1}$ King Abdullah University of Science and Technology (KAUST), KAUST Solar Center (KSC), Physical Sciences and Engineering Division (PSE), Thuwal 23955-6900, Kingdom of Saudi Arabia

${ }^{2}$ Instituto de Ciencia Molecular, Universidad de Valencia, C/ J. Beltrán 2, Paterna, Spain

$\dagger$ These authors contributed equally to this work.

Correspondence to: Email: erkan.aydin@kaust.edu.sa; stefaan.dewolf@kaust.edu.sa

Keywords: perovskites, silicon, tandem solar cells, textured, outdoor operation, temperaturedependent performance, bandgap

\begin{abstract}
Perovskite/silicon tandem solar cells promise power conversion efficiencies (PCE) beyond the Shockley-Queisser limit of single-junction devices, however, their actual outdoor performance is yet to be investigated. Here we fabricate $25 \%$-efficient two-terminal (2T) monolithic perovskite/silicon tandem solar cells and test them outdoors in a hot and sunny climate. We find that the temperature dependence of the bandgaps $\left(E_{\mathrm{g}}\right)$ of both silicon and perovskite - which follow opposing trends - shifts the devices away from current matching for $2 \mathrm{~T}$ tandems optimized at standard test conditions (STC, i.e., AM1.5G spectrum, $1000 \mathrm{~W} / \mathrm{m}^{2}, 25{ }^{\circ} \mathrm{C}$ ). Consequently, we argue that the optimal perovskite bandgap at STC is $E_{\mathrm{g}}<1.68 \mathrm{eV}$ (at STC) for field performance at operational temperatures $>55^{\circ} \mathrm{C}$, which is lower compared to earlier findings. This implies that
\end{abstract}


Br-lean perovskites with narrower bandgaps at STC - and, therefore, better phase stability - hold great promise for the commercialization of perovskite/silicon tandem solar cells.

\section{Main}

Metal halide perovskites have attracted significant attention for their application in tandem solar cells thanks to their high absorption coefficient, small Urbach energy, ${ }^{1,2}$ and tunability of their bandgap energy $\left(E_{\mathrm{g}}\right){ }^{3}$ Perovskite/silicon tandem solar cells are particularly appealing as they marry a proven high $\mathrm{PCE}^{4-9}$ - values $>29 \%$ have already been reported ${ }^{10}-$ with the industrial maturity of crystalline silicon (c-Si) photovoltaics (PV). ${ }^{11-13}$ In addition, unlike III-V/c-Si tandems, metal halide perovskites can be deposited by facile, versatile and potentially low-cost methods, even on macro-scale textured wafers, ubiquitous in c-Si PV manufacturing. ${ }^{5,8,14-16}$

The radiative-efficiency limit of perovskite/silicon tandems is $\sim 44 \%$ under STC. However, for monolithic, two-terminal (2T) tandems, this requires perfect current matching between the perovskite top cell and the c-Si bottom cell. For this, the perovskite $E_{\mathrm{g}}$ should be $\sim 1.73 \mathrm{eV}$ at STC (Supplementary Fig. 1a), ${ }^{11,17,18}$ which is significantly larger than the bandgaps typically used for record single-junction perovskite devices $(\sim 1.50-1.60 \mathrm{eV}) .{ }^{19-21}$ Conventionally, to open the $E_{\mathrm{g}}$ of halide perovskites, the Br:I halide ratio in the precursor (and thus the crystallized film) is increased ${ }^{22}$ However, beyond a Br:I ratio of $\sim 1: 4$ (corresponding to an $E_{\mathrm{g}}$ of $\sim 1.68 \mathrm{eV}$ ), I-rich and Br-rich domains segregate upon illumination, degrading device performance. ${ }^{23,24,25}$ Quite generally, the $E_{\mathrm{g}}$ of semiconductors also depends on temperature: with increasing temperature, it narrows for c-Si, but increases for perovskites. ${ }^{1,26}$ This is relevant for PV, as outdoor field-testing conditions (FTC) often imply elevated operating temperatures of PV modules well above $25{ }^{\circ} \mathrm{C}$, especially for high solar irradiation and space-constrained roof-top applications (due to heat- 
trapping effects) ${ }^{27}$ Therefore, what may be an ideal perovskite $E_{\mathrm{g}}$ for tandems at STC, is likely not an ideal $E_{\mathrm{g}}$ under actual FTC. Firstly, due to the red-shift of the c-Si $E_{\mathrm{g}}$ with temperature, the required perovskite $E_{\mathrm{g}}$ for current matching will already be lower than $1.73 \mathrm{eV}$. Secondly, due to the blue-shift of the perovskite $E_{\mathrm{g}}$ with temperature, its optimal $E_{\mathrm{g}}$ measured at STC will be even lower than that at FTC, impacting the design guidelines for efficient tandems.

In this work, we experimentally explore the impact of temperature-dependent changes of the $E_{\mathrm{g}}$ of perovskites and c-Si on $2 \mathrm{~T}$ tandem performance. To this end, we analyzed tandems with a certified PCE of $25 \%$, using double-side textured silicon heterojunction (SHJ) bottom cells, coated with perovskite top cells. Firstly, we experimentally measured the tandem temperature under actual outdoor conditions using field-test facilities at KAUST. With these data, we then calibrated a device-temperature model (details are given in the Methods section). By combining this model with relevant meteorological data, we simulated the tandem temperature over a complete year (2016), giving a realistic framework for tandem-performance analysis. Secondly, we experimentally characterized our tandems in a controlled laboratory environment under various temperatures and illumination spectra, with specific attention paid to their impact on current matching. Finally, to gain further insight, we developed a temperature-dependent radiativeefficiency model to support our findings. This modeling reveals that the optimal perovskite $E_{\mathrm{g}}$ for operation under FTC $>55^{\circ} \mathrm{C}$ is $<1.68 \mathrm{eV}$ (when measured under STC). Notably, such narrower$E_{\mathrm{g}}$ perovskites usually feature higher chemical stability. ${ }^{24,28}$ Overall, these findings provide a clear pathway towards commercial perovskite/silicon tandems that combine high performance with high stability. ${ }^{29-31}$ 


\section{Double-side textured monolithic perovskite/silicon tandems}

We fabricated monolithic $2 \mathrm{~T}$ tandems on double-side random-pyramid textured c-Si wafers. Such texturing effectively couples light into c-Si and is obtained with established industrial processes. SHJ technology was then used to fabricate the c-Si bottom cell, which was subsequently conformally coated by the perovskite top cell using the hybrid-deposition technique. ${ }^{5}$ Figs. 1a,b,c depict the geometry, cross-sections, and a photograph of the device, respectively. The top and bottom cells are electrically interconnected via a nano-crystalline silicon recombination junction, which combines a low tunnel resistance with a high shunt resilience and adequate coupling of nearinfrared light into the c-Si bottom cell. ${ }^{32}$

Fig. 1d shows the current density-voltage $(J-V)$ characteristics of our champion device with a certified PCE of $25.1 \%$ (steady-state maximum power point, MPP, of 25\%, at Fraunhofer ISE CalLab, over $0.8317 \mathrm{~cm}^{2}$ aperture area, see Supplementary Fig. 2). These results are consistent with our in-house $J-V$ measurements (Supplementary Fig. 3). The relatively narrow statistical distribution of the device parameters among independent tandem batches attests to the reproducibility of the sub-cell and tandem processing (Supplementary Fig. 4). We studied the spectral response of the tandems by external quantum efficiency (EQE) and 1-R measurements (R is reflectance, Fig. 1e). The tandems show $<2 \%$ average reflection in the range of $290-1100 \mathrm{~nm}$, instrumental ${ }^{33}$ in enabling of $19.8 \mathrm{~mA} \mathrm{~cm}^{-2}$ short-circuit current density $\left(J_{\mathrm{SC}}\right)$, which is one of the highest in literature (Supplementary Table 1). Between 1200-2500 nm, we find the average frontescape reflection (photons internally reflected off the rear SHJ c-Si contact and not absorbed) to be only $15 \%$ in our tandems; all other photons in this spectral range are absorbed in the opaque rear contact or by free carrier absorption in the front TCO, contributing to device heating. ${ }^{34}$ 


\section{Perovskite/silicon tandems under outdoor field conditions}

We first evaluate the tandem performance under direct sunlight and environmental factors, which is increasingly advocated as perovskite-based technologies progress towards market readiness. ${ }^{35}$ To protect the devices from environmental contaminants such as oxygen and water, the devices were vacuum laminated between glass and a polyolefin (POE) transparent back sheet. For details, see Methods and Supplementary Fig. 6a, b. As expected, the field-measured $J_{\mathrm{SC}}$ of the tandem follows the solar irradiation (Fig. 2a). We find that the $J_{\mathrm{SC}}$ drops by approximately $0.5 \mathrm{~mA} / \mathrm{cm}^{2}$ under 1-sun illumination when devices are encapsulated because of front-glass reflection (Supplementary Fig. 7). We also note that the solar spectrum measured at KAUST is relatively weak in the infrared, compared to the standard AM1.5G spectrum, as shown in Fig. 2c and Supplementary Fig. 8b. This will affect current matching for tandems optimized for STC, as detailed in Supplementary Note $1 .{ }^{29,36}$ The $V_{\mathrm{OC}}$ and $F F$ values reached their maximum at $~ 9: 30$ am (at about 0.5 suns) and maintain these values until $\sim 4: 30 \mathrm{pm}$. In the field, the maximum $V_{\mathrm{OC}}$ values are lower than those measured in the lab (Fig. 2b). This is partially due to the lower light intensity, but especially due to the device temperature (here encapsulated $T_{\text {cell }}$ is $\sim 50{ }^{\circ} \mathrm{C}$, and $\sim 60$ ${ }^{\circ} \mathrm{C}$ for un-encapsulated devices, see details in Supplementary Note 2). Quite generally, a high operating temperature is undesirable for solar cells as it reduces the PCE; the extent of which is expressed by the temperature coefficient (TC, defined as the change in PCE per temperature increment, normalized to the PCE at STC). Overall, we find that over the course of a given day, the energy yield of the tandem solar cell is mostly dictated by its $J_{\mathrm{SC}}$. Therefore, in this study we specifically explore the impact of temperature on the $J_{\mathrm{SC}}$ of $2 \mathrm{~T}$ tandem solar cells. 
To do so, we first estimated the expected $T_{\text {cell }}$ range under realistic operation conditions. For this, we fabricated $50 \mathrm{~cm}^{2}$ dummy tandems (without front metallization) with a thermocouple attached to their rear, followed by vacuum lamination between two glass sheets with a butyl rubber edge sealant (Supplementary Fig. 6b, d). These samples were exposed (under open-circuit conditions) to direct sunlight for a week in November 2019, at KAUST. For an ambient temperature ( $\left.T_{\mathrm{amb}} \max \right)$ of $38.4{ }^{\circ} \mathrm{C}, T_{\text {cell, }}$ max reached up to $60{ }^{\circ} \mathrm{C}$ (Fig. 2d). For these outdoor measurements, we used an open-rack test configuration (Supplementary Fig. 6c); significantly higher values for $T_{\text {cell }}$ can be expected in summer, and for roof-top installations because of heat-trap effects. ${ }^{37}$ Moreover, the device area here is much smaller than the encapsulation glass. Therefore, we expect that $T_{\text {cell }}$ in commercial-scale modules will be even higher than the reported values here, due to their significantly higher device packing densities.

These data, combined with local meteorological data, were then used as inputs to a thermal model (detailed in the Methods section), yielding simulated tandem temperatures over a complete year. Based on these calculations, a maximum perovskite/silicon $T_{\text {cell }}$ of $60{ }^{\circ} \mathrm{C}$ was predicted; $\sim 75^{\circ} \mathrm{C}$ appears, therefore, to be realistic for certain rooftop applications and/or under high solar irradiation..$^{27,38}$

\section{Temperature-dependent characteristics of the tandem cells}

Next, we performed temperature-dependent $J-V$ measurements in a controlled laboratory environment under a simulated AM1.5G spectrum, within the expected operational temperature range, by varying the temperature between 25 and $75^{\circ} \mathrm{C}$ (Fig. 3a). From these data, we calculated the TC for our tandem to be $-0.26 \% \mathrm{~K}^{-1}$ (see Supplementary Note 5). ${ }^{39}$ The c-Si bottom cell used in this study showed a TC of $-0.23 \% \mathrm{~K}^{-1}$ when normalized at $25^{\circ} \mathrm{C}$; a TC of $-0.26 \% \mathrm{~K}^{-1}-$ which is more typical for SHJ technology - was calculated when normalized at $40{ }^{\circ} \mathrm{C}$ (which is 
where the FF is linear with temperature; see Supplementary Fig. 20). ${ }^{39}$ Therefore, the addition of the perovskite top-cell barely affects the tandem TC. Notably, besides the physical factors that dictate the TC of single-junction solar cells, ${ }^{40}$ the TC of $2 \mathrm{~T}$ tandem solar cells is additionally affected by the possible current mismatch between the top and bottom cells. In the tandem under study here, we find this increasingly to be the case with temperature, resulting in a decreased $J_{\mathrm{SC}}$ of the device, thereby inflating the TC of the tandem. This current mismatch is due to the $E_{\mathrm{g}}$ shifts of c-Si and perovskite layers with temperature: the c-Si $E_{\mathrm{g}}$ narrows, but the perovskite $E_{\mathrm{g}}$ broadens with temperature. This implies that the TC of tandems can be minimized by optimizing the perovskite $E_{\mathrm{g}}$ towards current matching at the highest expected operational temperature. This possible mismatch-induced increase of the tandem TC is also of importance when comparing TCs of tandems as well as their PCEs at STC in the literature.

To further elucidate the $E_{\mathrm{g}}$ shift as a function of temperature, we studied the temperature-dependent

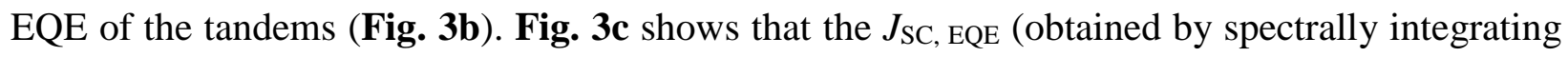
the product of EQE and incident solar spectrum) of the c-Si bottom cell increases with temperature. This is due to additional contributions of photons with longer wavelengths (as the c-Si $E_{\mathrm{g}}$ redshifts), as well as photons with shorter wavelengths (as the perovskite $E_{\mathrm{g}}$ blue shifts), coupling more light from two distinct spectral ranges in the c-Si cell underneath. Despite this, the overall $J_{\mathrm{SC}}$ of the $2 \mathrm{~T}$ tandem decreases because the $J_{\mathrm{SC}}$, EQE of the perovskite top cell decreases (as its $E_{\mathrm{g}}$ blueshifts), eventually limiting the overall $J_{\mathrm{SC}}$. Therefore, what may be the optimal perovskite $E_{\mathrm{g}}$ for STC (i.e., at $25^{\circ} \mathrm{C}$ ) may not be so for outdoor applications, where $T_{\text {cell }}$ is often significantly higher. 


\section{Impact of the spectral mismatch on the tandem performance}

To gain further insight into current mismatch in our tandem cells due to both variations in the solar spectrum and FTC operating temperature, we intentionally modified the incident spectrum of our solar simulator relative to the standard AM1.5G spectrum for device measurements at 25 and 75 ${ }^{\circ} \mathrm{C}$. In this way we mimic the effect of altering the perovskite $E_{\mathrm{g}}$ on the tandem $J_{\mathrm{SC}}$, which is a simple, yet powerful method to investigate current mismatch in $2 \mathrm{~T}$ tandems. ${ }^{41}$ Specifically, we altered the AM1.5G spectrum in our LED solar simulator by consecutively varying the blue (330$750 \mathrm{~nm}$ ) and red (751-1087 nm) parts of the spectrum, as shown in Fig. 4a, and Fig. 4b. We find that the $J_{\mathrm{SC}}$ of the tandem follows the same trend regardless of whether these blue or red parts were decreased, as illustrated in Fig. 4c. However, increasing these respective spectra above AM1.5G impacts the $J_{\mathrm{SC}}$ in opposite directions. Increasing the blue spectral part increased the $J_{\mathrm{SC}}$, suggesting that our tandem is slightly current mismatched at STC, caused by a current-limiting perovskite top cell. When, in addition to these spectral modifications, $T_{\text {cell }}$ was increased to $75^{\circ} \mathrm{C}$, the relative increase in the $J_{\mathrm{SC}}$ was more extensive, as now the $c$-Si subcell $J_{\mathrm{SC} \text {, EQE }}$ is also increased (due to the temperature-induced perovskite and c-Si $E_{\mathrm{g}}$ changes, as discussed above). Conversely, increasing the red spectral contribution slightly decreased the $J_{\mathrm{SC}}$ of the device. We also find that the current-dominating sub-cell always dictates the value of the tandem $F F$, as shown in Fig. 4c, which is in line with previous findings. ${ }^{41}$

To further maximize the tandem $J_{\mathrm{SC}}$, internal light trapping in the c-Si cell should be improved beyond the use of textured c-Si wafers, e.g., by suppression of parasitic infrared light absorption in its rear contact stack, as advocated for single-junction SHJ devices; ${ }^{33}$ the perovskite $E_{\mathrm{g}}$ should then be adapted accordingly. In addition, the temperature of the tandem cell should be considered 
when determining the optimal perovskite $E_{\mathrm{g}}$, to enable current matching and maximize the $J_{\mathrm{SC}}$ at FTC.

\section{Temperature-corrected bandgap of the perovskite top cells}

To further elucidate the effect of temperature on current matching in perovskite/silicon $2 \mathrm{~T}$ tandem solar cells, and provide tandem design guidelines with respect to the perovskite $E_{\mathrm{g}}$, we modeled their temperature-dependent radiative-efficiency limit based on the modeling methodology outlined by Jean et $a l .^{42}$ Our adapted model considers the effect of the temperature-dependent perovskite $E_{\mathrm{g}}$ and its Urbach energy on tandem performance; further details are given in the Methods. As discussed above, in the case of $2 \mathrm{~T}$ tandems, a departure from current-matching conditions leads to a significant drop in maximum PCE. In Supplementary Fig. 1a,b the traditional Shockley-Queisser efficiency limit (which only considers $E_{\mathrm{g}}$ values) for $4 \mathrm{~T}$ and $2 \mathrm{~T}$ perovskite/silicon tandems was calculated at $25{ }^{\circ} \mathrm{C}$ and is plotted as a function of the perovskite $E_{\mathrm{g}}$. In the $2 \mathrm{~T}$ case, a departure from the optimal, current-matched perovskite $E_{\mathrm{g}}$ of $1.73 \mathrm{eV}$ results in tandem power losses, due either to the perovskite or c-Si sub-cell limiting the $J_{\mathrm{SC}}$ (for a perovskite $E_{\mathrm{g}}$ lower or higher than $1.73 \mathrm{eV}$, respectively). This is not the case for the $4 \mathrm{~T}$ configuration since the sub-cells operate independently, and their output powers sum.

In general, the addition of band tailing in a semiconductor (caused by the structural and thermal disorder, expressed by the Urbach energy, $\left.E_{\mathrm{U}}\right),{ }^{2}$ tends to lower its corresponding efficiency limit by increasing the dark recombination current, and so decreasing the device voltage. Indeed, the voltage deficit $\left(E_{\mathrm{g}} / q-V_{\mathrm{OC}}, q\right.$ is the elementary charge $)$ in solar cells of different materials is known to show a strong correlation with $E_{\mathrm{U}} \cdot{ }^{1}$ Disorder - and thus $E_{\mathrm{U}}$ - scales with temperature; recently it was established that such changes correlate with temperature-induced losses in $V_{\text {OC. }}{ }^{1}$ Overall, it 
is the increase in the dark recombination current with temperature, to which band tailing is a contributor, that drives the absolute decrease in PCE values of both the $4 \mathrm{~T}$ and $2 \mathrm{~T}$ device configurations with increasing temperature.

Adding the effect of temperature on both $E_{\mathrm{g}}$ and $E_{\mathrm{U}}$ leads to a clear shift in the peak value of the optimal top cell $E_{\mathrm{g}}$ for $2 \mathrm{~T}$ tandems. This can be ascribed to the change in $E_{\mathrm{g}}$ of the two materials, the result of which is shown in the absorptance values used in the efficiency calculation shown in Fig. 5a,b. Note that we have assumed composition-independent values for both the perovskite's temperature-dependent $E_{\mathrm{U}}(\mathrm{T})$ and $E_{\mathrm{g}}(\mathrm{T})$ values. In the case of the $E_{\mathrm{g}}$, our temperature-dependent measurements on a variety of compositions are shown in Fig. 5c (in which the Br:I ratio is altered). The data shows the similar dependency of $E_{\mathrm{g}}$ over the temperature range investigated for all of the perovskite compositions, justifying our assumption in the model. ${ }^{1,2}$

For the $2 \mathrm{~T}$ case, we have also plotted the tandem $J_{\mathrm{SC}}$ as a function of the top cell $E_{\mathrm{g}}$, and include the current contributions of the individual sub-cells (dashed and dotted lines in the Fig. 5d). For any given temperature, the left side (lower values of perovskite $E_{\mathrm{g}}$ energies) of the peak in current denotes the values of the perovskite $E_{\mathrm{g}}$ that induce a constraint in the overall $J_{\mathrm{SC}}$ by the c-Si bottom cell. Here, the c-Si sub-cell is shaded by the top cell, and so the tandem $J_{\mathrm{SC}}$ follows the Si sub-cell current and its temperature dependence. On the right side of the apex, the wide- $E_{\mathrm{g}}$ perovskite current is constraining the device. The inversion in the trend of temperature at large perovskite $E_{\mathrm{g}}$ is due to the asymmetry in the trend of $E_{\mathrm{g}}$ with temperature, as also seen in the EQE (T) spectra of Fig. 3b. When the tandem is current limited by the bottom cell, the increase in temperature induces an increase in infrared absorption and so an increase in the overall $J_{\mathrm{SC}}$. The opposite is true when the device is constrained by the top cell: increasing the temperature results in lower currents in the top cell due to further broadening of the $E_{\mathrm{g}}$ away from the current-matching condition (Fig $\mathbf{5 d}$ ), as 
also demonstrated experimentally. By adding the effect of band tailing and light trapping to the radiative-efficiency limit calculations, we show that the ideal perovskite $E_{\mathrm{g}}$ value decreases substantially from the traditional calculation and that this value decreases further with increasing temperature (Fig. 5e). Critically, the optimal perovskite $E_{\mathrm{g}}$ value at the radiative limit decreases to below $1.68 \mathrm{eV}$ for relevant device operating temperatures. Indeed, by applying the model to the temperature and intensity data of Fig. $\mathbf{2 b}$, we calculate the top cell bandgap that delivers the highest energy yield at the radiative limit to be $\sim 1.685 \mathrm{eV}$ (see Supplementary Fig. 9). Such lower bandgaps are typically associated with materials with modest Br:I ratios, that are known to be more stable. In the case where the optimal top cell bandgap exceeds $1.68 \mathrm{eV}$, the relative power loss ceded by utilizing a perovskite of $1.68 \mathrm{eV}$ is likely minimal compared to previous determinations of the optimal efficiency, minimizing the incentive to explore wider bandgaps and so less stable perovskite compositions. Of course, these optimizations are sensitive to the input spectrum and will also be affected by parasitic losses in real cells and modules, as demonstrated in Supplementary Fig. 11. Note that for clarity, we display the data as a function of the top cell $E_{\mathrm{g}}$ at room temperature, though this value is also changing with temperature (Fig. 5f). 


\section{Conclusions}

We elucidated the temperature dependence of perovskite/silicon tandem solar cells, with specific attention to elevated temperatures typical of actual device operating conditions. To do so, we fabricated double-side textured monolithic perovskite/silicon tandem solar cells with a certified PCE of $25.1 \%$ and the highest $J_{\mathrm{SC}}$ value reported to date for such devices. We then studied the interplay between the operating temperature and the bandgaps of the sub-cells, and its effect on tandem performance. We tested these devices under both controlled lab conditions and outdoor field conditions. We find that, in a hot and sunny climate, perovskite/silicon tandem cells can easily reach $\sim 60{ }^{\circ} \mathrm{C}$ under direct sunlight and calculated that this can be as high as $\sim 75^{\circ} \mathrm{C}$ over the course of a year during operation.

These observations set a framework for the optimal design of perovskite/silicon-based tandem cells towards maximized power output under actual operating conditions. With increasing temperature, the $E_{\mathrm{g}}$ of $\mathrm{c}-\mathrm{Si}$ narrows, while for perovskites, it broadens, which affects currentmatching conditions. Therefore, for optimal tandem performance at field-testing conditions, we find that the perovskite $E_{\mathrm{g}}$ at standard test conditions $\left(\mathrm{STC}, 25^{\circ} \mathrm{C}\right.$ ) are lower compared to earlier findings. This conclusion is further supported by calculations using a modified temperaturedependent radiative-efficiency limit model. By applying this model, we find that the optimal perovskite $E_{\mathrm{g}}$ falls below $1.68 \mathrm{eV}$ (measured at $25^{\circ} \mathrm{C}$ ) at the radiative limit at elevated temperatures consistent with operating temperatures in a hot and sunny climate. This implies that conventional perovskites with moderate Br:I ratios, which do not suffer from halide segregation, can be used for tandems deployed in hot and sunny climates. Overall, our results give a straightforward perovskite-design framework for the development of stable and high-efficiency perovskite/silicon tandems for commercial deployment. 


\section{Acknowledgements}

The research reported in this publication was supported by funding from King Abdullah University of Science and Technology (KAUST) under award no. OSR-CARF URF/1/3079-3301 and award no. IED OSR-2019-4208. The authors thank TUV Rheinland Group, Germany, for providing solar spectra from their outdoor test field on the KAUST campus. J. Á. thanks the Spanish Ministry of Education, Culture and Sport for his pre-doctoral grant (FPU14/04466).

\section{Author contributions}

E.A and T.G.A. conceived the idea and designed the experiments. E.A. developed the perovskite top cells, top electrodes, and contact layouts and fabricated the tandem devices, E.A. and T.G.A. performed lab-based device characterizations. J. Á. contributed the fabrication of the perovskite absorber. T.G.A. developed nanocrystalline recombination junction and fabricated c-Si bottom cells. M.D.B. performed temperature-dependent absorptance measurements and prepared the silicon wafers. M.D.B and E.V.K. did the encapsulation of the devices. M.S. performed the field measurements. E.V.K. prepared the field data. L.X. performed the temperature prediction calculations. T.G.A. performed the temperature-dependent radiative-efficiency modeling and yield calculations. E.A., T.G.A., and S.D.W. composed the manuscript. All authors discussed the results, contributed to the writing, and commented on the manuscript.

\section{Competing Interests}

The authors declare no competing interests.

\section{Data Availability}

The datasets generated and analysed during the current study are available within the paper, its Supplementary Information and its Source Data files. 


\section{Methods}

\section{Fabrication of the silicon bottom cells}

SHJ bottom cells are fabricated on float-zone double-side-textured four inches wafers ( $n$-doped, resistivity 1-5 $\Omega . \mathrm{cm}$, thickness $250-280 \mu \mathrm{m}$ ). The texturing process was performed in an alkaline solution to achieve randomly distributed pyramids. The wafers were subsequently cleaned in RCA1 and RCA-2 solutions. Prior to the plasma enhanced chemical vapor depositions (PECVD), the wafers are dipped in a $5 \%$ hydrofluoric acid solution to remove the native oxide layer. The intrinsic, $n$ and $p$ amorphous silicon layers were deposited in a PECVD cluster (Indeotec Octopus II) with silane, hydrogen, phosphine, and trimethlyboron as input gases. The back electrode was formed by sequentially sputtering ITO and $\mathrm{Ag}$ (150 and $250 \mathrm{~nm}$, respectively) in the physical vapor deposition (PVD) part of the Octopus cluster. nc-Si:H(n)/nc-Si:H(p) recombination junction is formed by the sequential deposition of the n- and p-doped silicon films utilizing high hydrogen: silicon gas flow ratios. All depositions were performed at temperatures of $\leq 200{ }^{\circ} \mathrm{C}$.

\section{Fabrication of the perovskite top cells}

To achieve $p-i-n$ configuration perovskite top cells, $12 \mathrm{~nm}$ of spiro-TTB (>99\%, Lumtec) was deposited by Angstrom EvoVac thermal evaporation system (base pressure $<1 \times 10^{-6}$ Torr, evaporation rate of $0.25 \AA / s$, quartz crucible) as the HTL on top of nc-Si:H(n)/nc-Si:H(p) recombination junction. On top, as the first step of the sequential method, $\mathrm{PbI}_{2}(>99.99 \%$, beads from Alfa Aesar) and $\mathrm{CsBr}(>99.9 \%$, Sigma-Aldrich) were co-evaporated using the same evaporation system (base pressure $<1 \times 10^{-6}$ Torr, evaporation rate of $1 \AA \mathrm{s}^{-1}$ for $\mathrm{PbI}_{2}$ and of $0.1 \AA^{-1}$ for $\mathrm{CsBr}$, aluminum oxide crucibles). During the evaporation processes, no intentional heating or cooling was applied to the substrates. Later, as the second step of the process, a precursor 
of formamidinium iodide and bromide (in 3 to 1 molar ratio, $0.6 \mathrm{M}$ in ethanol, Greatcell Solar) was spin-casted onto the co-evaporated layer in a nitrogen glove box (spin speed of $4000 \mathrm{rpm}$ during $35 \mathrm{~s}$, quenching after $5^{\text {th }}$ second). Then, the resultant intermediate phase is annealed at $150{ }^{\circ} \mathrm{C}$ for $30 \mathrm{~min}$ in a controlled humidity environment (30\%) to form the perovskite absorber. 20 $n m \mathrm{C}_{60}$ was evaporated within the same system (base pressure $<1 \times 10^{-6}$ Torr, evaporation rate of $0.2 \AA / \mathrm{s}$, quartz crucible). $20 \mathrm{~nm}$ of $\mathrm{SnO}_{2}$ was deposited by the atomic layer deposition technique using a Picosun system at $100{ }^{\circ} \mathrm{C}$ substrate temperature using tetrakis(dimethylamino)tin (TDMASn) and $\mathrm{H}_{2} \mathrm{O}$ as precursors (kept in $80{ }^{\circ} \mathrm{C}, 134$ cycles). To form the transparent electrodes, $100 \mathrm{~nm}$ of indium zinc oxide (IZO) was sputtered in an Angstrom Engineering system using a 3inch target $\left(90 \% \mathrm{In}_{2} \mathrm{O}_{3} / 10 \% \mathrm{ZnO}\right)$ with a radio frequency power of $42 \mathrm{~W}$ (base pressure $<1 \times 10^{-6}$ Torr). The four-point probe measured sheet resistance of the IZO films was $40 \Omega / \mathrm{sq}$ (for $100 \mathrm{~nm}$, on glass). Silver metal fingers $(200 \mathrm{~nm})$ were thermally evaporated through the shadow masks with a rate of $2.5 \AA^{-1}$. As a final step, $100 \mathrm{~nm}$ of $\mathrm{MgF}_{2}$ antireflection films were thermally evaporated within the same system (random sized crystals from Plasmaterials, >99.9\%, $1 \AA \mathrm{s}^{-1}$, base pressure of $<1 \times 10^{-6}$ Torr). Here, all reported thickness values represent the thickness values on flat substrates. However, for the thermal evaporation onto the textured interfaces, a 1.7 geometric factor ratio (1.7 times thicker) is applied during the deposition.

\section{Fabrication of the various bandgap of perovskite films with the one-step process}

$\mathrm{PbI}_{2}$ and $\mathrm{PbBr}_{2}$ (Alfa Aesar), methylammonium bromide (Greatcell Solar), formamidinium iodide (Greatcell Solar), and cesium iodide (Sigma Aldrich) were dissolved in a mixture of DMF: DMSO (4:1, Sigma Aldrich) to prepare the perovskite precursor solution. Thin films of perovskites $\left(\mathrm{FACsMAPbI}_{3-\mathrm{x}} \mathrm{Br}_{\mathrm{x}}\right)$ with different Bromide content $(6 \%, 15 \%, 20 \%, 30 \%$ of the total halide content) were deposited in nitrogen atmosphere via spin coating at two-step spin process (first 
2000 and later $5000 \mathrm{rpm}$ ) on soda-lime glass substrates, using chlorobenzene as a solvent quencher. Subsequently, the films were annealed on a hotplate at $100{ }^{\circ} \mathrm{C}$ for $10 \mathrm{~min}$.

\section{Temperature-dependent bandgap measurements}

Carry 5000 (Agilent Technologies) UV-Vis tool was used to measure the absorptance of the fabricated perovskite films. The band gap values of the perovskites with different bromide content at different temperatures. The temperature control was provided via a Janis cryostat (with quartz window) with Peltier heaters and under a nitrogen flushing.

\section{Characterization of the photovoltaic performances}

Room temperature and temperature-dependent $J-V$ measurements were performed using Wavelabs Sinus 220 LED-based solar simulator with AM 1.5G irradiance spectrum. The light intensity was calibrated using Fraunhofer ISE CalLab certified c-Si solar cells. The temperature is controlled during the measurement using a vacuum stage using a Peltier element. Standard $J-V$ measurements were performed at $25^{\circ} \mathrm{C}$. The illumination area of the devices was $1.03 \mathrm{~cm}^{2}$ which is determined by the laser cut shadow mask coated with matte black paint. The device measurements were performed at $200 \mathrm{mV} / \mathrm{s}$ scan speed using Keithley 2400 source meter. The stabilized power measurements (at $25{ }^{\circ} \mathrm{C}$ ) were recorded via the homemade LabView ${ }^{\circledR}$ based software using a three-point perturbation method. Room temperature and temperature-dependent $\mathrm{EQE}$ measurements were performed using PV-Tools LOANA equipment by measuring the temperature from the backside of the wafers. When measuring perovskite top cells, the tandem devices were light-biased by IR LEDs $(930 \mathrm{~nm})$; when measuring c-Si bottom cells, the tandem devices were light-biased by a blue LED $(450 \mathrm{~nm})$ to saturate the subcells. To enable the near short-circuit conditions for each subcell, MPP voltages were applied on the devices. For in-house 
measurements, all device characterizations were performed at ambient air with average $\mathrm{RH} \sim 50 \%$ and without any encapsulation.

\section{Outdoor measurements}

For the field measurements, two sets of samples were prepared. For temperature tracking of the devices, monolithic perovskite/silicon tandem solar cells (without metal grids), single-junction semitransparent perovskite solar cells (without metal grids), and single junction SHJ cells were prepared over $50 \mathrm{~cm}^{2}$ area to mimic the realistic applications. All these devices were encapsulated between two glasses (1 mm low iron glasses) with $10 \mathrm{~mm}$ wide butyl rubber edge sealants, which were laminated at $100{ }^{\circ} \mathrm{C}$. The butyl rubber sealants were located $3 \mathrm{~cm}$ away from the wafers to avoid additional heating from the sealants. The temperature values were recorded directly from the bottom surface of the wafers using PT100 thermocouples and thermal paste. For power tracking, perovskite/silicon tandem solar cell samples were encapsulated with glass/POE/glass structure at $140{ }^{\circ} \mathrm{C}$ using Ecolam vacuum laminators. To avoid the contribution of the SHJ bottom cell to the current generation, the rest of the active area is shaded by non-transparent aluminum tapes.

Photovoltaic outdoor data was acquired using an I-V tracer from EKO (model MP-160) in November 2019. The I-V characteristics and temperature of multiple samples were probed successively using the multiplexers MI-520 and MI-540, respectively (all from EKO). The power values were tracked with a time interval of $10 \mathrm{~min}$ and with $200 \mathrm{mV} / \mathrm{s}$ scan speed identical to laboratory measurements. Current-voltage curves were measured with a scan rate of $200 \mathrm{mV} / \mathrm{s}$. The electronic equipment is kept inside a cooled house at $23^{\circ} \mathrm{C}$. Data acquisition was performed with a computer running EKO's MP160 software. The global horizontal irradiance on the plane of the devices (mounted on the same structure as the devices) was measured using the pyranometer MS- 
802 (EKO). The solar cells were mounted on a structure with a tilt angle of $25^{\circ}$ and South orientation. The structure is located in the outdoor testing field on the KAUST campus, near the village of Thuwal, Saudi Arabia (coordinates 22.302494, 39.110737).

\section{Temperature-dependent bandgap modeling}

To model the temperature dependence of PCE we followed the modified detailed balance methodology in ref. ${ }^{42}$ which incorporates exponential Urbach band tailing effects into the radiative limit efficiency calculation first established by Shockley and Queisser. ${ }^{43}$ Instead of assuming an abrupt, step-function at band edge, subgap absorption, defined by the Urbach energy of the absorber material, is added, as in ref. ${ }^{42}$. We apply the same approach to calculate the radiative efficiency limit of perovskite/silicon tandem cells using previously measured temperaturedependent data for the Urbach energy of both materials. ${ }^{1}$ In addition, we measure the $E_{\mathrm{g}}$ of the perovskite under study and use well-established parameterizations for the temperature dependence

of the c-Si $E_{\mathrm{g}}{ }^{44}$ to establish a temperature-dependent radiative efficiency limit model for the perovskite/silicon tandem cell architecture. As in the original calculation of Shockley and Queisser, we assume ideal absorption above the $E_{\mathrm{g}}$ with no reflection losses. To model the subgap absorption, in addition to the temperature-dependent Urbach energy, we assume a single pass in the perovskite top cell and Lambertian light trapping with ideal rear reflection in the c-Si bottom cell.

\section{Module temperature prediction modeling}

The empirical equation ${ }^{45}$ below is used for simulating the device operating temperature $T_{m}$.

$$
T_{m}=E \times e^{a+b \times v}+T_{a}
$$


Where $T_{a}$ is the ambient temperature, $E$ is the sun irradiance, $v$ is the wind speed, $a$ and $b$ are empirically determined coefficients. We firstly use the experimental measured outdoor data in November 2019 at Jeddah to fit the parameters $a$ and $b$. Using the empirical model with the localized fitted parameters, the device temperature for the complete year of 2016 in Jeddah was simulated based on the environment data from photovoltaic geographical information system. ${ }^{46}$ 


\section{References}

1 Ledinsky, M. et al. Temperature dependence of the urbach energy in lead iodide perovskites. J. Phys. Chem. Lett. 10, 1368-1373 (2019).

2 De Wolf, S. et al. Organometallic halide perovskites: sharp optical absorption edge and its relation to photovoltaic performance. J. Phys. Chem. Lett. 5, 1035-1039 (2014).

3 McMeekin, D. P. et al. A mixed-cation lead mixed-halide perovskite absorber for tandem solar cells. Science 351, 151-155 (2016).

4 Jošt, M. et al. Textured interfaces in monolithic perovskite/silicon tandem solar cells: Advanced light management for improved efficiency and energy yield. Energy Environ. Sci. 11, 3511-3523 (2018).

5 Sahli, F. et al. Fully textured monolithic perovskite/silicon tandem solar cells with $25.2 \%$ power conversion efficiency. Nat. Mater. 17, 820-826. (2018).

6 Shen, H. et al. In situ recombination junction between p-Si and $\mathrm{TiO}_{2}$ enables highefficiency monolithic perovskite/Si tandem cells. Sci. Adv. 4, eaau9711 (2018).

$7 \quad \mathrm{Xu}$, J. et al. Triple-halide wide-band gap perovskites with suppressed phase segregation for efficient tandems. Science 367, 1097-1104 (2020).

8 Hou, Y. et al. Efficient tandem solar cells with solution-processed perovskite on textured crystalline silicon. Science 367, 1135-1140 (2020).

9 Kim, D. et al. Efficient, stable silicon tandem cells enabled by anion-engineered widebandgap perovskites. Science 368, 155-160 , (2020).

10 NREL, Best Research-Cell Efficiency Chart. https://www.nrel.gov/pv/assets/images/efficiency-chart.png (accessed May 2020).

11 Leijtens, T., Bush, K. A., Prasanna R., McGehee M. D. Opportunities and challenges for tandem solar cells using metal halide perovskite semiconductors. Nat. Energy 3, 828-838 (2018).

12 Allen, T. G., Bullock, J., Yang, X., Javey. A., De Wolf, S. Passivating contacts for crystalline silicon solar cells. Nat. Energy, 4, 914-928 (2019).

13 Haegel, N. M. et al. Terawatt-scale photovoltaics: Transform global energy. Science 364, 836-838 (2019).

14 Battaglia, C., Cuevas, A., De Wolf, S. High-efficiency crystalline silicon solar cells: status and perspectives. Energy Environ. Sci. 9, 1552-1576 (2016).

15 Sansoni, S. et al. Eco-friendly spray deposition of perovskite films on macroscale textured surfaces. Adv. Mater. Technol. 5, 1901009 (2020).

16 Chen, B. et al. Blade-coated perovskites on textured silicon for $26 \%$-efficient monolithic perovskite/silicon tandem solar cells. Joule 4, 850-864 (2016).

17 Eperon, G., Hörantner, M. T., Snaith, H. J. Metal halide perovskite tandem and multiplejunction photovoltaics. Nat. Rev. Chem. 1, 0095 (2017). 
18 Albrecht, S., Rech, B. Perovskite solar cells: On top of commercial photovoltaics. Nat. Energy 2, 1-2 (2017).

19 Jiang, Q. et al. Surface passivation of perovskite film for efficient solar cells. Nat. Photonics 13, 460-466. (2019).

20 Yoo, J. J. et al. An interface stabilized perovskite solar cell with high stabilized efficiency and low voltage loss. Energy Environ. Sci. 12, 2192-2199 (2019).

21 Zheng, X. et al. Managing grains and interfaces via ligand anchoring enables 22.3\%efficiency inverted perovskite solar cells. Nat. Energy 5, 131-140 (2020).

22 Bush, K. A. et al. Compositional engineering for efficient wide band gap perovskites with improved stability to photoinduced phase segregation. ACS Energy Lett. 3, 428-435, (2018).

23 Mahesh, S. et al. Revealing the origin of voltage loss in mixed-halide perovskite solar cells. Energy Environ. Sci. 13, 258-267 (2020).

24 Correa-Baena, J.-P. et al. Promises and challenges of perovskite solar cells. Science 358, 739-744 (2017).

25 Aydin, E., De Bastiani, M., De Wolf, S. Defect and contact passivation for perovskite solar cells. Adv. Mater. 0, 1900428 (2019).

26 Singh, P., Ravindra, N. M. Temperature dependence of solar cell performance-an analysis. Sol. Energy Mater. Sol. Cells 101, 36-45 (2012).

27 Sample, T., Virtuani, A. Modification to the standard reference environment (SRE) for nominal operating cell temperature (NOCT) to account for building integration. Proc. of the 24th EU-PVSEC, Hamburg, 3332 (2009).

28 Hoke, E. T. et al. Reversible photo-induced trap formation in mixed-halide hybrid perovskites for photovoltaics. Chem. Sci. 6, 613-617 (2015).

29 Horantner, M. T., Snaith, H. Predicting and optimising the energy yield of perovskiteon-silicon tandem solar cells under real world conditions. Energy Environ. Sci. 10, 19831993.(2017).

30 Jacobs, D. A. et al. Light management: A key concept in high-efficiency perovskite/silicon tandem photovoltaics. J. Phys. Chem. Lett. 10, 3159-3170 (2019).

31 Dupré, O., Niesen, B., De Wolf, S., Ballif, C. et al. Field performance versus standard test condition efficiency of tandem solar cells and the singular case of perovskites/silicon devices. J. Phys. Chem. Lett. 9, 446-458 (2018).

32 Sahli, F. et al. Improved optics in monolithic perovskite/silicon tandem solar cells with a nanocrystalline silicon recombination junction. Adv. Energy Mater. 8, 1701609 (2017).

33 Holman, Z. C. et al. Infrared light management in high-efficiency silicon heterojunction and rear-passivated solar cells. J. App. Phys. 113, 013107 (2013).

34 Silverman, T. J. et al. Reducing operating temperature in photovoltaic modules. IEEE J. Photovoltaics 8, 532-540 (2018). 
35 Khenkin, M. V. et al. Consensus statement for stability assessment and reporting for perovskite photovoltaics based on ISOS procedures. Nat. Energy 5, 35-49 (2020).

36 Tress, W. et al. Performance of perovskite solar cells under simulated temperatureillumination real-world operating conditions. Nat. Energy 4, 568-574 (2019).

37 Kurnik, J., Jankovec, M., Brecl, K., Topic, M. Outdoor testing of PV module temperature and performance under different mounting and operational conditions. Sol. Energy Mater. Sol. Cells 95, 373-376 (2011).

38 Nordmann, T., Clavadetscher, L. in Proceedings of $3^{\text {rd }}$ World Conference on Photovoltaic Energy Conversion 3, 2243-2246 (2003).

39 Haschke, J. et al. The impact of silicon solar cell architecture and cell interconnection on energy yield in hot \& sunny climates. Energy Environ. Sci. 10, 1196-1206 (2017).

40 Dupré, O., Vaillon, R., Green, M. A. Physics of the temperature coefficients of solar cells. Sol. Energy Mater. Sol. Cells 140, 92-100 (2015).

41 Köhnen, E. et al. Highly efficient monolithic perovskite silicon tandem solar cells: analyzing the influence of current mismatch on device performance. Sustainable Energy Fuels 3, 1995-2005 (2019).

42 Jean, J. et al. Radiative efficiency limit with band tailing exceeds $30 \%$ for quantum dot solar cells. ACS Energy Lett. 2, 2616-2624 (2017).

43 Shockley, W., Queisser, H. J. Detailed balance limit of efficiency of p-n junction solar cells. J. App. Phys. 32, 510-519 (1961).

44 Green, M. A. Intrinsic concentration, effective densities of states, and effective mass in silicon. J. App. Phys. 67, 2944-2954 (1990).

45 King, D. L., Boyson, W. E., Kratochvill, J. A. Photovoltaic array performance model. Sandia Report, SAND2004-3535 (United States. Department of Energy, 2004).

46 Photovoltaic Geographical Information System, https://re.jrc.ec.europa.eu/pvg tools/en/tools.html (accessed January 2020). 


\section{Figure Captions}

Fig. 1. Design and performance of the perovskite/silicon tandem solar cells. a, Schematic illustration, b, SEM cross-sectional view, and c, photograph of the double-side-textured tandem solar cells prepared in this work with a hybrid-processed perovskite absorber layer. The detailed contact layout is given in Supplementary Fig. 5. d, $J-V$ characteristics of the certified champion device which were measured at Fraunhofer ISE CalLab. The inset in Fig. d shows a light-beaminduced current (LBIC) image under $532 \mathrm{~nm}$ excitation, demonstrating the uniformity of the perovskite top cell. The color bar shows the signal intensity. The perovskite absorber layer has a $E_{\mathrm{g}}$ of $1.63 \mathrm{eV}$. e) The corresponding EQE and 1-R data for the same device shown in (d) at 25 ${ }^{\circ} \mathrm{C}$ overlaid on the AM1.5 solar spectral irradiance. The given $J_{\mathrm{SC}}$ values represent the integrated values from EQE measurements of each subcell. f, Photovoltaic parameters of a perovskite/silicon tandem under MPP tracking conditions for 5 min.

Fig. 2. Outdoor performance of perovskite/silicon tandem solar cells. a, Photovoltaic parameters of a representative perovskite/silicon tandem solar cell tested outdoors at KAUST during a week in November 2019, and respective solar irradiance as well as ambient and dummy device temperatures (active area of $50 \mathrm{~cm}^{2}$ without a front electrode). Note that the $J_{\mathrm{SC}}$ of the tandem device is calculated based on the aperture opening on the encapsulation glass, which was $1.17 \mathrm{~cm}^{2}$. PD represents the power density. The decrease of the $F F$ values are assigned to contact degradation as discussed in Supplementary Note 3. b, Zoom-in of the data highlighted in Fig. 2a (day 2). The dashed line in the Suns data represent the measured Suns values. The orange shaded values represent the interpolated data, free of shading. The intensity fluctuations in the measured Suns data are due to local shading on the pyranometer. The sharp decrease in device temperature at 11:00 am is due to an uptake of the wind speed (as shown in Supplementary Note 4), resulting in a slight PCE increase, underlining the effectiveness of convective cooling. The PCE values were calculated by dividing the power density by the Suns data. c, Spectral power over one day measured at KAUST on November $7^{\text {th }}, 2019$ along with the suns data in Fig. 2b. d, Ambient temperature, and predicted cell temperature values for a perovskite/silicon tandem device over a complete year (based on weather data of 2016, KAUST). The tabulated data for d are given in Supplementary Fig. 18b. The day in each month with the highest peak temperature values is displayed. The orange lines represent the average peak temperature of the month. The given temperature values in $\mathrm{d}$ represents the $24 \mathrm{~h}$ time frame for each day.

Fig. 3. Temperature-dependent performance of the perovskite/silicon tandem solar cells. Temperature-dependent a, $J-V$ (R: reverse, F: forward scan) and b, EQE and 1-R (R is reflectance) characteristics of perovskite/silicon tandem devices. The bottom of Fig. $3 \mathrm{~b}$ shows a zoom-in into the region of the band edge for both subcells. c, Temperature-dependent change of EQE measured current density $\left(J_{\mathrm{SC}, \mathrm{EQE}}\right)$ values for both sub-cells together with the $J-V$ measured current density $\left(J_{\mathrm{SC}}\right)$ of a tandem solar cell, showing current limiting conditions. 
Fig. 4. The performance of the tandem solar cells under current mismatch conditions. a, The generated spectrum of the different blue $(330-750 \mathrm{~nm})$ and red $(751-1087 \mathrm{~nm})$ regions together with standard AM1.5G spectra and b, corresponding integrated photon flux values for the spectrum generated via an LED-based solar simulator. Here, $1000 \mathrm{~W} \mathrm{~m}^{-2}$ denotes the standard AM1.5G spectrum. Red and blue dashed lines in panel $\mathbf{b}$ are a guide to the eye showing the blue $(330-750 \mathrm{~nm})$ and red $(751-1087 \mathrm{~nm})$ portion of the photon flux for the spectral ratio of 1, generated by the LED. c, The change of the device characteristics for various "blue" and "red" spectrum fractions for 25 and $75^{\circ} \mathrm{C}$. The intensity of the spectrum fractions are shown in panel $\mathrm{b}$ for each red and blue combination. Here "spectrum fraction" defines the intensity of the spectrum (for the given range) normalized to the intensity of LED-generated AM1.5G standard spectrum. $75^{\circ} \mathrm{C}$ is chosen as a representative of elevated temperatures.

Fig. 5. The calculated temperature-dependent performance of the ideal perovskite/silicon tandem solar cells. The simulated band edge absorptance for $\mathbf{a}, \mathrm{c}-\mathrm{Si}$ and $\mathbf{b}$, perovskite semiconductors considering the temperature dependencies of $E_{\mathrm{U}}$ (Urbach energy) and $E_{\mathrm{g}}$ (bandgap energy). Note the simulated perovskite absorptance data represents an RT $E_{\mathrm{g}}$ of 1.66 $\mathrm{eV}$, and the slope of the $E_{\mathrm{g}}$ change was $0.31 \mathrm{meV} \mathrm{K}^{-1}$ as calculated from c, the experimentally acquired UV-vis-NIR absorbance of $20 \% \mathrm{Br}$ content in the mixed halide perovskite films deposited on glass substrates as shown in Supplementary Fig. 22. The slope of the $E_{\mathrm{g}}$ change for $\mathrm{Si}$ was $-0.273 \mathrm{meV} \mathrm{K}^{-1}$. The dotted lines are guides to the eye. $\mathbf{d}$, The variation of the total $2 \mathrm{~T}$ tandem cell current as a function of the top cell perovskite $E_{\mathrm{g}}$ and cell temperature. The dotted lines show the current contribution from the perovskite subcell, while the dashed lines show the contribution from the c-Si subcell. The maximum efficiency occurs when the two values are equal. e, Corresponding PCE values for both 2T (solid lines) and 4T (dotted lines) configurations as a function of top cell $E_{\mathrm{g}}$ and temperature. The temperature legend in Fig. 5a, b also applies to panels $\mathrm{d}$ and e. f. The calculated ideal room temperature $E_{\mathrm{g}}$ of the top cell perovskite absorber at different operating temperatures. The dotted lines are guides to the eye. 

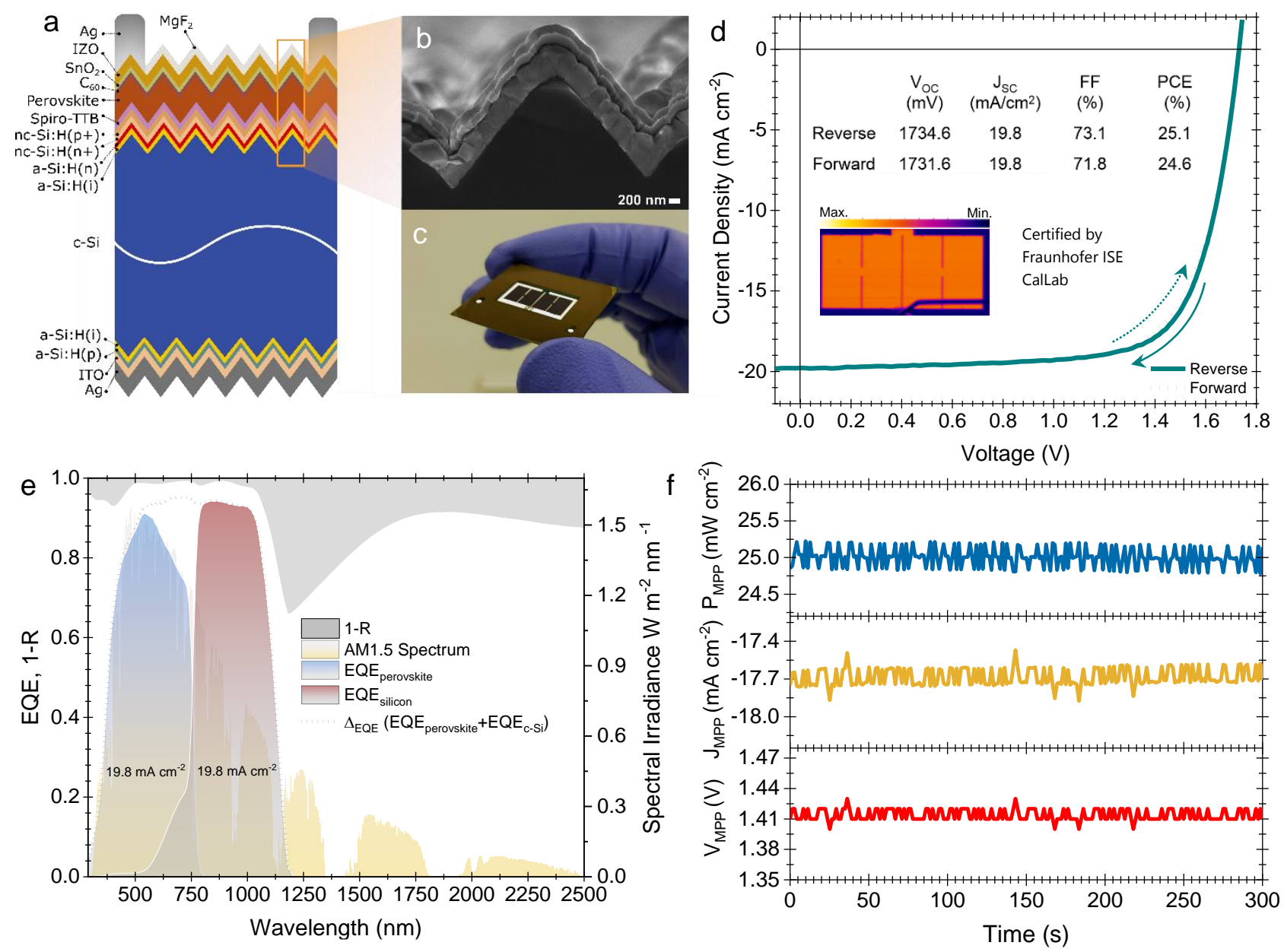

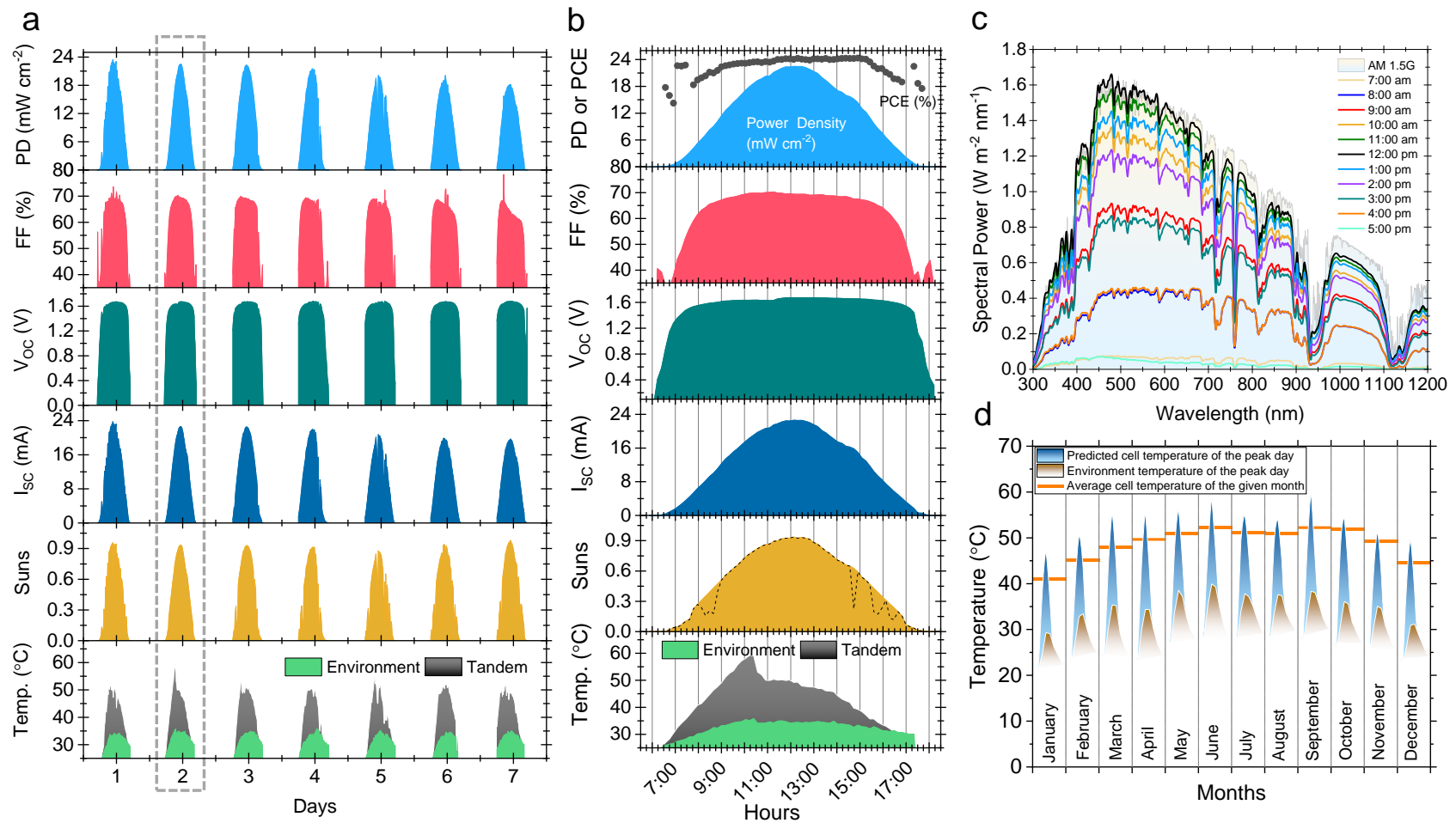

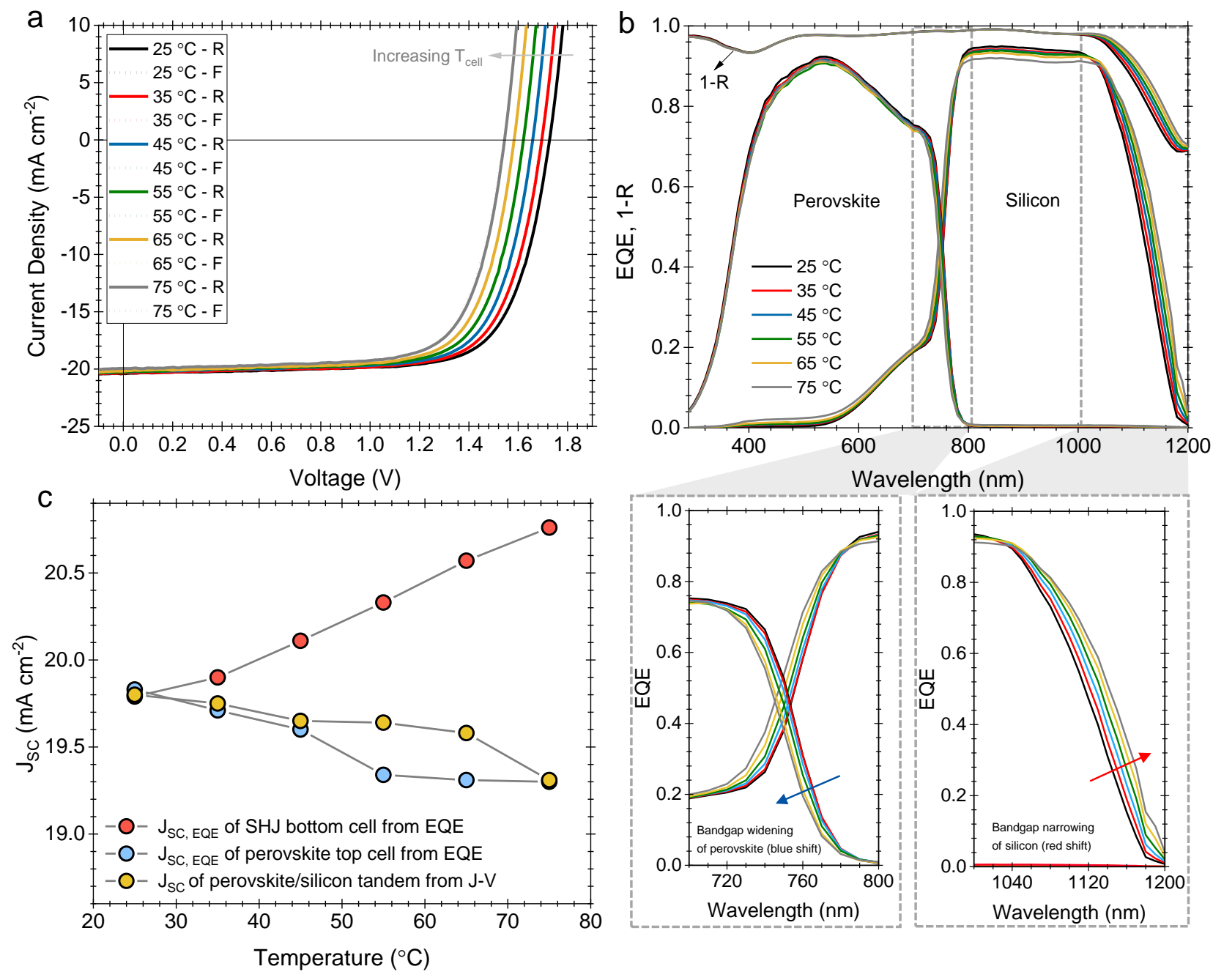

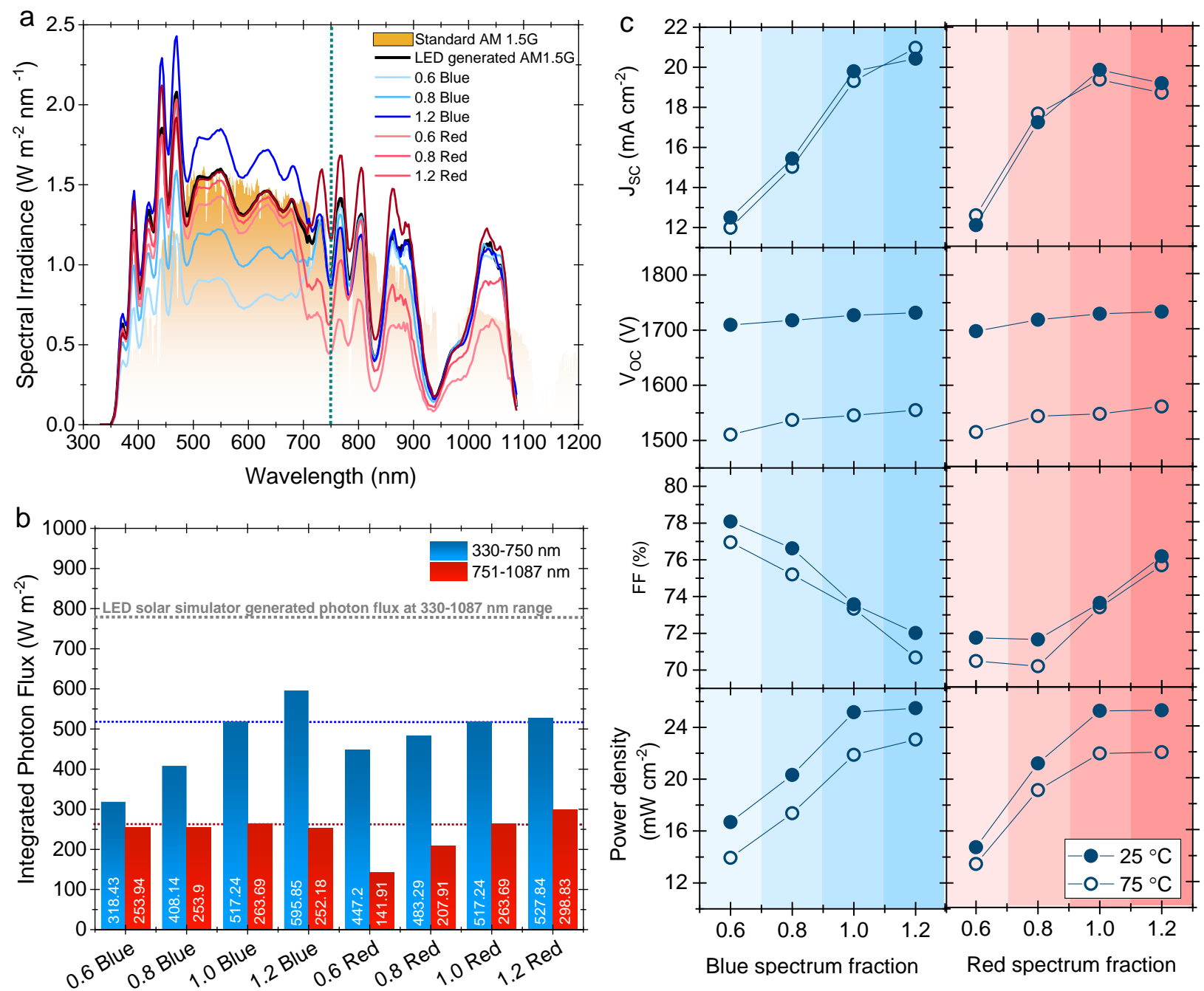

Blue spectrum fraction

Red spectrum fraction 

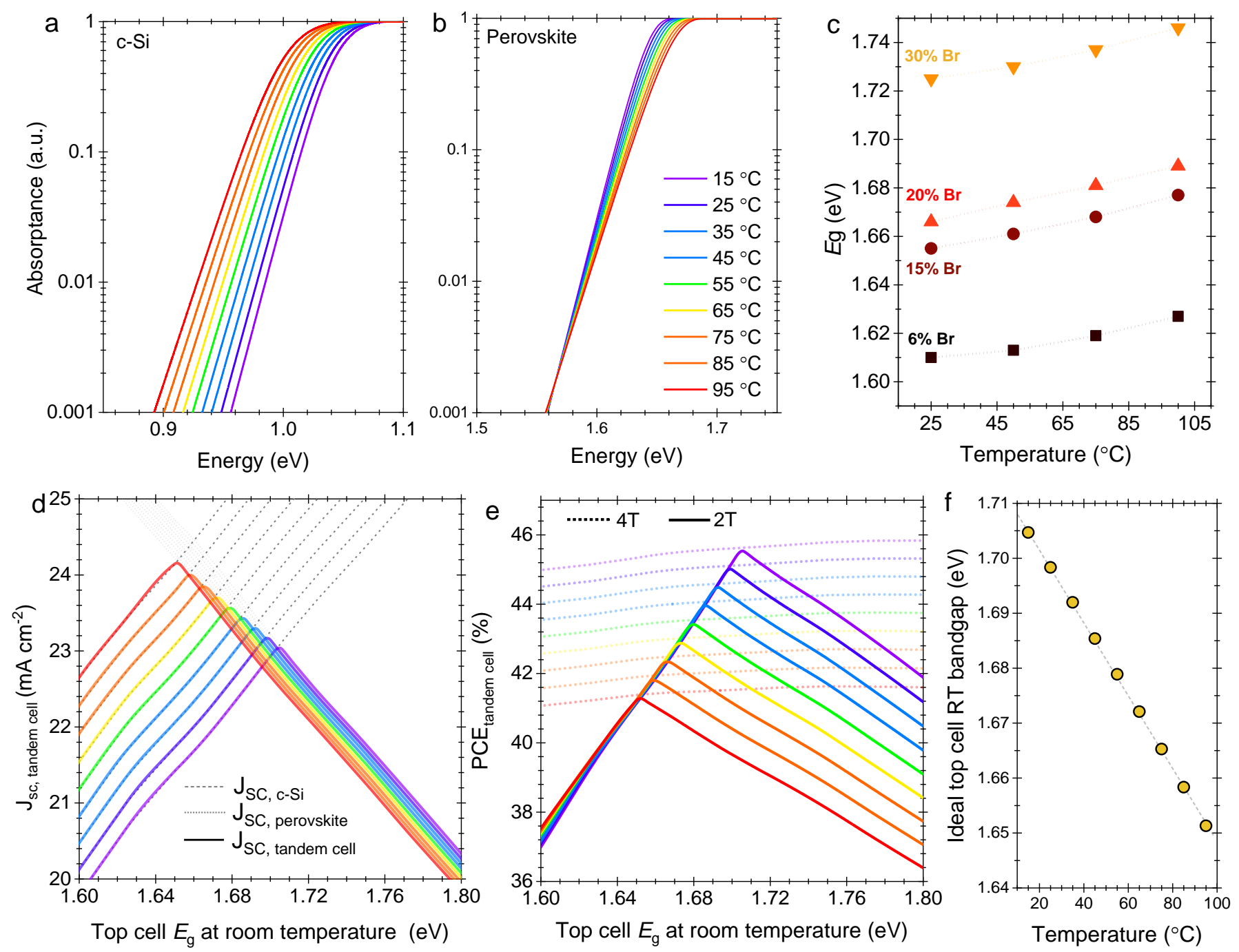